\title{
HIPK2 and cancer cell resistance to therapy
}

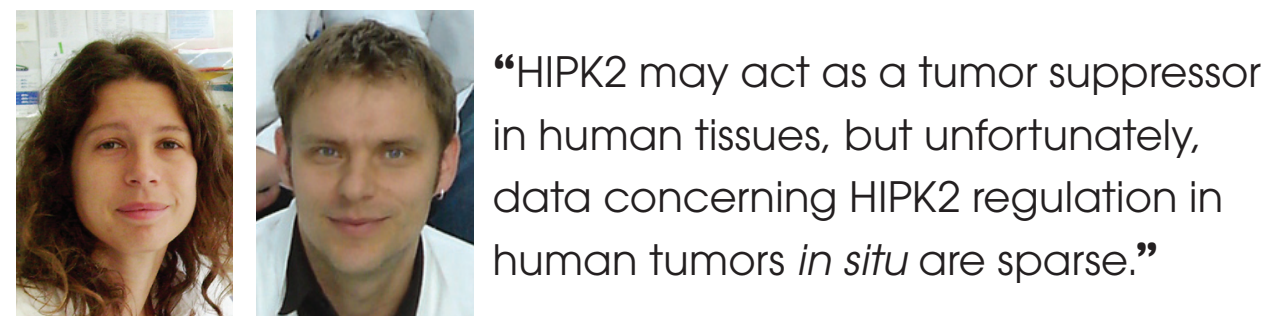

Eva Krieghoff-Henning \& Thomas $G$ Hofmann ${ }^{\dagger}$

†Author for correspondence: Thomas G Hofmann = Cellular Senescence A210, German Cancer Research Center, Im Neuenheimer Feld 242, 69120 Heidelberg, Germany = Tel. : +496221424631 = Fax: +496221 424902 -.t.hofmann@dkfz.de

Within the last decade, members of the homeodomain-interacting protein kinase (HIPK) family of serine/threonine kinases have been found to regulate cell-fate decisions at multiple levels. The best-studied family member is HIPK2, which was originally identified as a transcriptional corepressor of homeodomain transcription factors such as NK-3 [1].

HIPK2 is now considered as a central switch in the targeting of cells towards apoptosis upon genotoxic stress by phosphorylating tumor suppressor p53 at Ser46 [2,3]. This modification promotes $\mathrm{p} 53$ acetylation by CREB-binding protein and redirects the transcription factor p53 to proapoptotic target genes such as PUMA and Noxa.

This pathway has received much attention during the last few years, and it has been demonstrated that HIPK2 facilitates p53-dependent apoptosis in tumor cell lines in response to UV- and ionizing radiation [4], as well as to a variety of chemotherapeutic agents, namely cisplatin [5], adriamycin [6] and roscovitine [7].

However, importantly, the proapoptotic functions of HIPK2 are not limited to the p53 pathway. HIPK 2 also plays a role in TGF- $\beta$ signaling, where it is important for JNK activation and JNK-mediated apoptosis [8]. Moreover, it seems that HIPK2 can facilitate the degradation of the antiapoptotic transcriptional corepressor $\mathrm{C}$-terminal binding protein (CtBP). This degradation is dependent on Ser 422 phosphorylation of $\mathrm{CtBP}$, which may occur directly via HIPK2 itself [9], or indirectly via JNK [10], and, again, takes place after cytotoxic insults, such as UV irradiation or cisplatin treatment of tumor cell lines. Very recently, HIPK2 has also been reported to antagonize $\mathrm{LEF} / \beta$-catenin signaling, possibly by facilitating the degradation of $\beta$-catenin via an as yet unknown mechanism, which might antagonize the growth of colorectal carcinoma and hepatoma cells. This $\beta$-catenin degradation may be in part responsible for the capacity of HIPK2 to downregulate VEGF expression, and thus to reduce neoangiogenesis. Intriguingly, HIPK2 is bound and activated by Axin, a scaffold protein found to enhance $\beta$-catenin degradation, and an axin-HIPK2-p53 complex was reported to activate proapoptotic p53 target genes [11]. Taken together, these findings suggest that HIPK2 may act as a tumor suppressor in human tissues through different pathways, preventing aberrant proliferation of severely damaged cells by driving them into apoptosis.

Unfortunately, data concerning HIPK2 regulation in human tumors in situ are sparse. The earliest study that deals with HIPK2 levels in human cancer reports downregulation of HIPK2 in many thyroid and breast carcinomas in comparison with the respective normal tissues [12]; however, these interesting findings still need to be extended by functional studies. Several years later in 2007, two missense mutations were identified within the HIPK2 speckle retention signal in human acute myeloblastic leukemias (AMLs) and in myelodysplastic syndrome, which led to an aberrant diffuse distribution of HIPK2 within the cell nucleus [13]. The disturbed localization of HIPK2 led to impaired transcription by the AML1 and p53 transcription factors. However, aberrantly expressed HIPK2 was only found at a very low frequency (two out of 130 samples), and thus, a putative causative role of HIPK2 in leukemic cell transformation remains to be shown. However, interestingly, in a very recent publication, Wee $e t$ al. report that a potentially leukemogenic fusion protein consisting of PEBP $2 \beta$ (also termed CBF $\beta$ ) and SMMHC, relocalizes HIPK2 to filamentous structures in the cytoplasm, thereby inhibiting

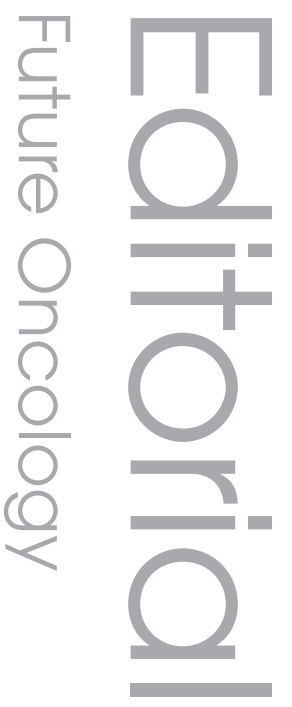

\section{future ${ }_{\text {medicine }}^{\text {siss }}$ fs}


HIPK2-dependent phosphorylation of p300 in the nucleus [14]. Similarly, Pierantoni et al. demonstrated in 2007 that the expression of the oncogenic high-mobility group A1 (HMGA1) protein in breast carcinoma is well correlated with increased cytoplasmic HIPK2 staining and decreased apoptosis in these tumors, likely via decreased p53 Ser46 phosphorylation [15]. In addition, in 2007, Wei et al. published an interesting study in which $H I P K 2^{-1-}$ and even $H I P K 2^{+/-}$mice exhibited higher sensitivities to skin tumor formation in a two-step carcinogenesis model, indicating that in normal mouse skin, HIPK2 indeed suppresses malignant cell growth [16]. With regard to HIPK2 levels in tumors, it is particularly interesting to note that the malignant phenotype already appeared in heterozygous mice, which supports the notion that HIPK2 dosage is crucial in suppressing aberrant cell growth, and suggests HIPK2 as a haploinsufficient tumor suppressor.

However, by contrast, HIPK2 does not seem to suppress, but rather to promote tumor growth in cervical cancer, since it was strongly expressed in more than $75 \%$ of cervical cancer samples as compared with only $10 \%$ of normal control tissues in another very recent study [17]. The expression of HIPK2 in this cancer type apparently correlated with tumor progression. Moreover, another recently published observation of Deshmukh et al. suggests that in certain cancers, enhanced HIPK2 expression is actually advantageous for tumor progression [18]: HIPK2 is overexpressed in approximately two-thirds of pilocytic astrocytomas, which are relatively benign gliomas that primarily occur in childhood and early adolescence, and to a certain (albeit much lower) extent in other glioma types [18].

\section{"HIPK2 may well turn out to be a promising target for tumor therapy."}

Unfortunately, neither of these studies went on to investigate the relationship between HIPK2 expression and sensitivity towards chemo- or radiotherapy. Therefore, until recently, it remains to be shown that the HIPK2 status does indeed have an impact on the outcome of cancer therapy. Owing to the functional promiscuity of HIPK2, it is entirely possible that HIPK2 deficiency or proficiency will differentially influence the sensitivity to irradiation and cytotoxic chemicals in p53positive and -negative malignancies. In $\mathrm{p} 53$ expressing cells, HIPK2 activation would be expected to potently enhance apoptosis in response to treatment. Thus, we would expect that in p53-positive cancer, HIPK2 expression would be selected against. Indeed, Puca $e t$ al. reported earlier this year that overexpression of HIPK2 in p53 wild-type, chemoresistant ovarian cancer cells can render these cells sensitive to therapy by mediating p 53 phosphorylation [19]. Puca et al. also presented a second study suggesting that HIPK2 is required for native folding of the $\mathrm{p} 53$ protein in colon cancer cells; surprisingly, HIPK2 action can be replaced by the addition of zinc to the cells, possibly suggesting a therapeutic function of zinc ions in p53-proficient malignancies [20]. However, in p53-negative cancer, HIPK2 may still promote apoptosis by derepressing CtBP target genes or by inducing JNK, but recent evidence from HIPK 2 knockout mice and the studies of Al-Beiti et al. and Deshmukh et al. suggest that HIPK2 may also play a role in cell proliferation and survival in some cell types $[17,18]$. Indeed, this was shown by using the glioma cell-line U87, which exhibits enhanced cell growth in a colony formation assay when transfected with wild-type, but not kinase dead HIPK2 [18]. Furthermore, a prosurvival role of HIPK2 in dopaminergic neurons was also reported using a HIPK2 knockout mouse model [21]. Thus, it is conceivable that some cancer patients might even benefit from HIPK2 inhibition, particularly in cases of elevated HIPK2 levels in the absence of $\mathrm{p} 53$.

The regulation of HIPK2 levels has been subject to intense investigation in previous years. We and others have shown that HIPK2 is an unstable protein that is degraded via the proteasomal pathways. In particular, the ubiquitin ligases WSB-1 [22] on the one hand and Siah-1 and -2 on the other hand have been shown to target HIPK2 for degradation, a process that is inhibited by cytotoxic stress, and in the case of Siah-1, via the activation of the DNA damage checkpoint kinases ATM and/or ATR [23]. This implies that in order to block HIPK2 function, cancer cells may not only upor downregulate HIPK2 on the mRNA level, but may induce its stabilization or degradation by modulating its ubiquitin ligases. Indeed, we could demonstrate that depletion of Siah-1 leads to an enhanced apoptosis induction [23]. Intriguingly, it has been shown that Siah proteins are active in hypoxia, which is often present in solid tumors [24]. Siah-1 also appears to be a p53 target gene, implying that Siah-1 is part of a negative-feedback mechanism that 
helps cells to survive after p53 induction. Concordantly, p53-deficient tumor cell lines indeed show elevated HIPK2 protein levels [23], suggesting that HIPK2 may be stabilized in p53-deficient cancer.

Interestingly, a recent study suggests that Siah proteins are essential mediators of oncogenic rasinduced cell transformation and tumorigenesis, but whether HIPK2 degradation is important in this transformation has not yet been determined [25].

In summary, HIPK2 may well turn out to be a promising target for tumor therapy. Exogenous activation of HIPK2 may sensitize particularly p53-proficient cancer cells to conventional chemotherapy, whereas inhibition of HIPK2 in tumors of neuronal origin may inhibit growth or even induce apoptosis in these cells. Importantly, HIPK2 levels may constitute a useful prognostic marker for the outcome of conventional chemotherapy, but much work remains to be carried out in order to systematically characterize the cancer entities in which HIPK2 modulates cell death or survival.

\section{Acknowledgments}

We apologize to all colleagues who made important contributions that could not be cited here due to space limitations.

\section{Financial \& competing interests disclosure}

The authors have no relevant affiliations or financial involvement with any organization or entity with a financial interest in or financial conflict with the subject matter or materials discussed in the manuscript. This includes employment, consultancies, honoraria, stock ownership or options, expert testimony, grants or patents received or pending, or royalties.

No writing assistance was utilized in the production of this manuscript.

\section{Bibliography}

Papers of special note have been highlighted as: - of interest

1. Kim YH, Choi CY, Lee SJ, Conti MA, Kim Y: Homeodomain-interacting protein kinases, a novel family of co-repressors for homeodomain transcription factors. J. Biol. Chem. 273, 25875-25879 (1998).

2. Hofmann TG, Moller A, Sirma $\mathrm{H}$ et al.: Regulation of $\mathrm{p} 53$ activity by its interaction with homeodomain-interacting protein kinase-2. Nat. Cell Biol. 4, 1-10 (2002).

- Elucidates the central mechanism by which HIPK2 regulates cell fate - that is, the induction of p53-dependent apoptosis.

3. D'Orazi G, Cecchinelli B, Bruno T et al.: Homeodomain-interacting protein kinase-2 phosphorylates p 53 at Ser 46 and mediates apoptosis. Nat. Cell Biol. 4, 11-19 (2002).

- Elucidates the central mechanism by which HIPK2 regulates cell fate - that is, the induction of p53-dependent apoptosis.

4. Dauth I, Kruger J, Hofmann TG: Homeodomain-interacting protein kinase 2 is the ionizing radiation-activated p53 serine 46 kinase and is regulated by ATM. Cancer Res. 67, 2274-2279 (2007).

5. Di Stefano V, Rinaldo C, Sacchi A, Soddu S, D’Orazi G: Homeodomain-interacting protein kinase- 2 activity and $\mathrm{p} 53$ phosphorylation are critical events for cisplatin-mediated apoptosis. Exp. Cell Res. 293, 311-320 (2004).
6. Gresko E, Roscic A, Ritterhoff S, Vichalkovski A, Del Sal G, Schmitz ML: Autoregulatory control of the $\mathrm{p} 53$ response by caspase-mediated processing of HIPK2. EMBO J. 25, 1883-1894 (2006).

7. Wesierska-Gadek J, Schmitz ML, Ranftler C: Roscovitine-activated HIP2 kinase induces phosphorylation of wt p53 at Ser-46 in human MCF-7 breast cancer cells. J. Cell. Biochem. 100, 865-874 (2007).

8. Hofmann TG, Stollberg N, Schmitz ML, Will H: HIPK2 Regulates transforming growth factor- $\beta$-induced c-Jun $\mathrm{NH}(2)$ terminal kinase activation and apoptosis in human hepatoma cells. Cancer Res. 63, 8271-8277 (2003).

9. Zhang Q, Yoshimatsu Y, Hildebrand J, Frisch SM, Goodman RH: Homeodomain interacting protein kinase 2 promotes apoptosis by downregulating the transcriptional corepressor CtBP. Cell 115, 177-186 (2003).

10. Wang SY, Iordanov M, Zhang Q: c-Jun $\mathrm{NH} 2$-terminal kinase promotes apoptosis by down-regulating the transcriptional co-repressor CtBP. J. Biol. Chem. 281, 34810-34815 (2006).

11. Rui Y, Xu Z, Lin S et al.: Axin stimulates $\mathrm{p} 53$ functions by activation of HIPK2 kinase through multimeric complex formation. EMBO J. 23, 4583-4594 (2004).

12. Pierantoni GM, Bulfone A, Pentimalli F et al:: The homeodomain-interacting protein kinase 2 gene is expressed late in embryogenesis and preferentially in retina, muscle, and neural tissues. Biochem. Biophys. Res. Commun. 290, 942-947 (2002).
13. Li XL, Arai $\mathrm{Y}$, Harada $\mathrm{H}$ et al:: Mutations of the HIPK2 gene in acute myeloid leukemia and myelodysplastic syndrome impair AML1- and p53-mediated transcription. Oncogene 26(51), 7231-7239 (2007).

14. Wee HJ, Voon DC, Bae SC, Ito Y: PEBP $2 \beta / C B F \beta$-dependent phosphorylation of RUNX1 and p300 by HIPK2: implications for leukemogenesis. Blood 112(9), 3777-3787 (2008).

15. Pierantoni GM, Rinaldo C, Mottolese M et al: : High-mobility group A1 inhibits $\mathrm{p} 53$ by cytoplasmic relocalization of its proapoptotic activator HIPK2. J. Clin. Invest. 117, 693-694 (2007).

16. Wei G, Ku S, Ma GK et al.: HIPK2 represses beta-catenin-mediated transcription, epidermal stem cell expansion, and skin tumorigenesis. Proc. Natl Acad. Sci. USA 104, 13040-13045 (2007).

17. Al-Beiti MA, Lu X: Expression of HIPK2 in cervical cancer: correlation with clinicopathology and prognosis. Aust. NZ J. Obstet. Gynaecol. 48, 329-336 (2008).

18. Deshmukh H, Yeh TH, Yu J et al:: High-resolution, dual-platform aCGH analysis reveals frequent HIPK2 amplification and increased expression in pilocytic astrocytomas. Oncogene 27(34), 4745-4751 (2008).

19. Puca R, Nardinocchi L, Pistritto G, D'Orazi G: Overexpression of HIPK2 circumvents the blockade of apoptosis in chemoresistant ovarian cancer cells. Gynecol. Oncol. 109, 403-410 (2008). 
20. Puca R, Nardinocchi L, Gal H et al.: Reversible dysfunction of wild-type p 53 following homeodomain-interacting protein kinase-2 knockdown. Cancer Res. 68 , 3707-3714 (2008).

21. Zhang J, Pho V, Bonasera SJ et al.: Essential function of HIPK2 in TGF $\beta$-dependent survival of midbrain dopamine neurons. Nat. Neurosci. 10, 77-86 (2007).

22. Choi DW, Seo YM, Kim EA et al.: Ubiquitination and degradation of homeodomain-interacting protein kinase 2 by WD40 repeat/SOCS box protein WSB-1. J. Biol. Chem. 283, 4682-4689 (2008).

- Provides important insight into the regulation of the HIPK2 protein itself.
23. Winter M, Sombroek D, Dauth I et al.: Control of HIPK2 stability by ubiquitin ligase Siah-1 and checkpoint kinases ATM and ATR. Nat. Cell Biol. 10, 812-824 (2008).

- Provides important insight into the regulation of the HIPK2 protein itself.

24. Nakayama K, Frew IJ, Hagensen M et al.: Siah2 regulates stability of prolylhydroxylases, controls HIF1 $\alpha$ abundance, and modulates physiological responses to hypoxia. Cell 117, 941-952 (2004).

25. Schmidt RL, Park CH, Ahmed AU et al.: Inhibition of RAS-mediated transformation and tumorigenesis by targeting the downstream E3 ubiquitin ligase seven in absentia homologue. Cancer Res. 67, 11798-11810 (2007).

\section{Affiliations}

- Thomas G Hofmann Cellular Senescence A210, DKFZ-ZMBH Alliance, German Cancer Research Center, Im Neuenheimer Feld 242, 69120 Heidelberg, Germany Tel.: +49622 1424631 Fax: +496221424902 t.hofmann@dkfz.de

Eva Krieghoff-Henning Cellular Senescence A210, DKFZ-ZMBH Alliance, German Cancer Research Center, Im Neuenheimer Feld 242, 69120 Heidelberg, Germany Tel.: +496221424914 Fax: +49622142 4902 e.krieghoff@dkfz.de 